\title{
A Fuzzy Scheme for Gait Cycle Phase Detection Oriented to Medical Diagnosis
}

\author{
Mario I. Chacon-Murguia, Omar Arias-Enriquez, and Rafael Sandoval-Rodriguez \\ Visual Perception Applications on Robotic Lab, Chihuahua Institute of Technology, Mexico \\ \{mchacon, oarias, rsandova\} @itchihuahua.edu.mx
}

\begin{abstract}
Gait cycle phase detection provides useful information to diagnose possible problems on walking. The work reported here proposes the analysis of gait kinematic signals, extracted from videos, through fuzzy logic to automatically determine the different phases in the human gait cycle. The function of the fuzzy system is to detect the gait phases, loading response, mid-stance, terminal stance, pre-swing, initial swing, mid-swing, and terminal swing, using 2D information from a sagittal plane. The system was tested with normal and non-normal gait cycles. Experimental findings proved that the fuzzy detection system is able to correctly locate the phases using only 2D information. The maximum phase timing shift error generated was $2 \%$. Thus, it may be concluded that the proposed system can be used to analyses gait kinematic and detect gait phases in normal cycle and absences of them in non-normal cycles. This information can be considered for gait anomaly detection and therapeutic purposes.
\end{abstract}

Keywords: gait phase analysis, fuzzy systems, video segmentation.

\section{Introduction}

Nowadays, it is well known that vision systems have increased their contribution in areas related to human motion analysis. One of these areas is human gait analysis which is related to the style or characteristics involved in a person's walking [1]. Gait analysis has proved to be relevant to several fields, including biomechanics, robotics, sport analysis, rehabilitation engineering, etc. [2-5]. In the medical field gait analysis has been oriented to medical diagnosis where different types of application have been developed [6]. A specific area in medical application is human movement analysis, where systems are designed to perceive and analyze human movement of upper and lower limbs for the purpose of providing diagnosis and/or therapy [7]. Gait analysis is a paramount aid for therapist because they can analyze different gait pathologies and thus determine appropriate therapies for their patients [8]. These pathologies may be a consequence of spinal cord lesion, stroke, multiple sclerosis, rheumatoid arthritis etc.

Currently, there exist different systems, with advantages and disadvantages, which perform gait dynamic or kinematic quantifications. Dynamic evaluations are achieved through accelerometers, force platforms, etc. The kinematic quantification is done by electrogoniometers and 2D and 3D vision systems [9],[10]. However, not many are related to detect gait phase detection oriented to medical diagnosis. The aim of this paper is to present the development of a fuzzy system to automatically detect gait 
phases based on the analysis of information provided by a non-invasive vision system [11]. There are some works related to this kind of systems like the one reported in [12] which uses signal from the sagittal plane like, hip, knee and ankle. Another work [13] rely on $3 \mathrm{D}$ information. In our work it is proved that a $2 \mathrm{D}$ system can be used instead a $3 \mathrm{D}$, and that the input variables generated with the $2 \mathrm{D}$ system are suitable for gait phase detection. The information used in the proposed system corresponds to the kinematic of the sagittal plane of the knee, hip, and variables time and stage.

The organization of the paper is structured as follows. Section 2 provides the definition of gait cycle and its phases. The development of the proposed system is explained in Section 3. Experimental tests of the system are presented in Section 4 and the results and conclusions are commented in section 5.

\section{Gait Cycle Definition}

Human walking is a locomotion method which involves the use of lower limbs allowing the displacement of the body under a stable state. In this process, one lower limb serves as the support, meanwhile the other one provides propulsion. The cycle of a human gait is divided in two main periods; the stance which takes approximately $60 \%$ of the cycle and the swing that covers the remaining time. In turn, the stance period is divided into the phases: Loading response, $L R$, mid-stance, $M S_{s}$, terminal stance, $T S_{s}$, and pre-swing, $P S_{w}$. The swing period is composed of: Initial swing, $I S$, mid-swing, $M S_{w}$, and terminal swing, $T S_{w}$. Table 1 shows the different gait phases and their corresponding timing and cycle percentages [1].

Table 1. Normal gait phase timing

\begin{tabular}{|c|c|c|}
\hline Phase number & Phase & Percentage of phase occurrence in full cycle \\
\hline 1 & Loading response & $0-10 \%$ \\
\hline 2 & Mid-stance & $10-30 \%$ \\
\hline 3 & Terminal stance & $30-50 \%$ \\
\hline 4 & Pre-swing & $50-60 \%$ \\
\hline 5 & Initial swing & $60-73 \%$ \\
\hline 6 & Mid-swing & $73-87 \%$ \\
\hline 7 & Terminal swing & $87-100 \%$ \\
\hline
\end{tabular}

\section{Fuzzy System Scheme for Gait Phase Detection}

The inputs to the system correspond to sagittal information related to knee, hip, time and an extra input called stage. The variable time is related to the gait cycle percentage. The information of these variables is acquired by an image processing method described in [14] which is a method to obtain this information without using artificial marks. The system can be formalized as follows. Given kinematic signals of the knee and hip, $x_{k}(n), x_{h}(n)$, derive two time variables $x_{T}(n)$ and $x_{S}(n)$ to design the mapping

$$
F:\left\{x_{k}(n), x_{h}(n), x_{T}(n), x_{S}(n)\right\} \Rightarrow P
$$

where $x_{k}(n), x_{h}(n), x_{T}(n), x_{s}(n)$ and $P$ will be fuzzified and will have the next fuzzy values 


$$
\begin{aligned}
& x_{k}(n)=\left\{x_{\text {kLow }}, x_{\text {kHigh }}\right\} \\
& x_{h}(n)=\left\{x_{\text {hLow }}, x_{\text {hMedium }}, x_{\text {hHigh }}\right\} \\
& x_{T}(n)=\left\{x_{\text {TLow }}, x_{\text {TMedium }}, x_{T H i g h}\right\} \\
& x_{S}(n)=\left\{x_{S L R}, x_{S M S}, x_{S S S}\right\} \\
P= & \left\{P_{L R}, P_{M S S}, P_{T S S}, P_{S w}, P_{I S w}, P_{M S w}, P_{T S w}\right\}
\end{aligned}
$$

The mapping $F$ is accomplished by a Mamdani type fuzzy system through the implication relation

$$
R\left(\left\{x_{k}(n), x_{h}(n), x_{T}(n), x_{S}(n)\right\}, P\right)
$$

\subsection{Input and Output Variables and Their Fuzzy Definition}

The knee information is obtained from an average knee kinematic signal shown in Figure 1a. Its membership functions represents the different intervals in the $y$ axis. The trajectory can be divided into two intervals; low $x_{k \text { Low }}$, and high $x_{k H i g h}$. Figure $1 \mathrm{~b}$ corresponds to an average hip movement and it can be divided in three intervals, $x_{h}$ Low $, x_{h \text { Medium }}, x_{h \text { High }}$. The intervals for the input variable time are also low, medium and high as shown in Figure 2. The membership functions for the input variable stage are not determined based on intervals because it is used to distinguish the phases loading response and initial swing and between mid-stance and terminal swing. They are defined based on phase occurrence during the gait cycle.
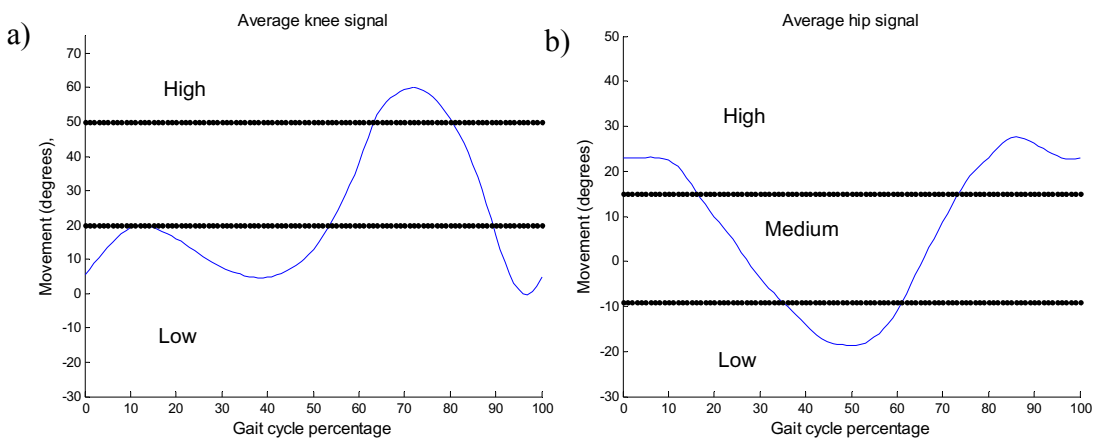

Fig. 1. a) Grade movement intervals of an average knee signal. b) Grade movement intervals of an average hip signal.

It is noteworthy that the inputs to the fuzzy systems need to be normalized, where zero correspond to the minimum value and 1 to the maximum. The membership functions, MFs, of the input variables are shown in Figure 3. The function parameters, mean $\mu$ and variance $\sigma$ are as follow. For the hip input the gaussians are $G_{\text {Low }}(0$, $0.175), G_{\text {Medium }}(0.5,0.175), G_{H i g h}(1,0.175)$. For the knee the MFs are similar except the value medium is not used. The gaussian parameters for the input time are $G_{\text {Low }}(0,0.256), G_{\text {Medium }}(0.5,0.175)$ and $G_{\text {High }}(1,0.175)$. As commented before the 


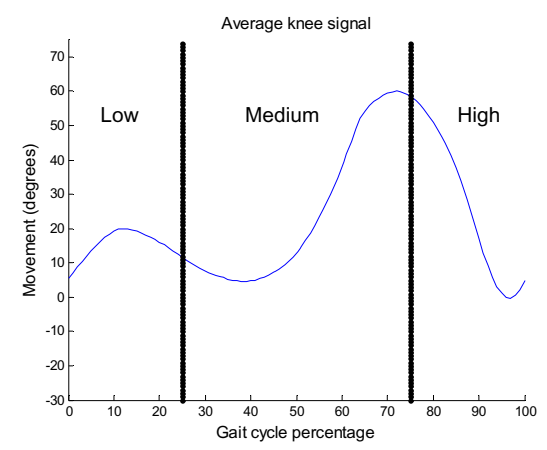

Fig. 2. Time intervals of an average knee signal

input stage is incorporated to the system in order to distinguish between the phases loading response and initial swing and between mid-stance and terminal stance. This is because some inference rules are fired for similar conditions. Thereby, the variable stage allows the differentiation among those fired rules so the correct output is obtained from the system. The stage variable is in fact other time variable with three possible fuzzy values loading response, $x_{L R}$, mid-stance $x_{M S s}$, and swing - stance $S S$. The function $x_{S L R}$ is used to differentiate between loading response and mid-swing and it is represented through a $Z$ function with parameters $Z_{L R}(0.1,0.11)$. The function $x_{S M S}, G_{M S}(0.208,0.05)$ has the purpose to distinguish between mid-stance and terminal stance. Finally the function $x_{S S S}$, represented by a $S$ function $S(0.405,0.632)$, is used to resolve between the swing and stance phases.

The gait phases, defined in Section 2, correspond to the values of the output variable Phase of the system. These values are represented by triangular membership functions with the following parameters and illustrated in Figure 4. $P_{L R}(0,1,2), P_{M S s}(1,2,3)$, $P_{T S s}(, 2,3,4), P_{P S w}(3,4,5), P_{I S}(4,5,6), P_{M S w}(5,6,7), P_{T S w}(6,7,8)$.

\subsection{Fuzzy System Rules for Phase Detection}

The fuzzy system designed is a Mamdani system defined by rules

$$
R_{i}: \text { if } x_{1} \text { is } A_{i 1} \mathbf{A N D}, \ldots, x_{n i} \text { is } A_{i n} \text { THEN } \mathrm{y}_{i} \text { is } B
$$

The defuzzification scheme used was the mean of maximum. The rules are determined by an intuitive reasoning. That is, the output is just the consequence of a spe-

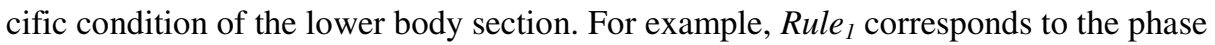
loading response, $L R$, and it is derived by knowing that in a normal gait cycle the position of the hip is approximately 20 degrees. If we locate this value for the average hip movement, Figure 1b, we found that correspond to a value of High. In the case of the knee during the same phase, it has a low value for the intervals defined in Figure 1a. 
a)

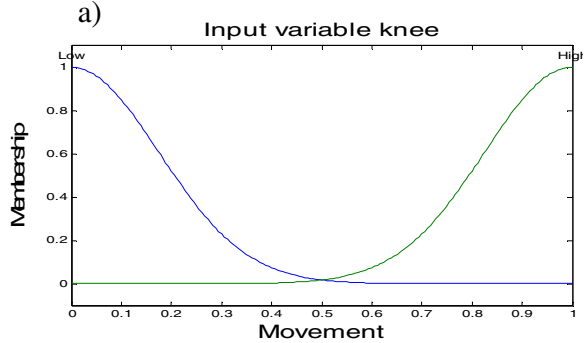

c)

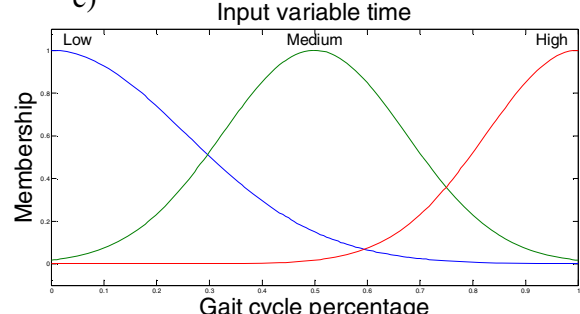

b)

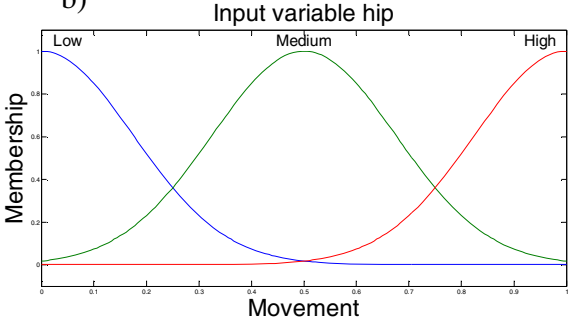

d)

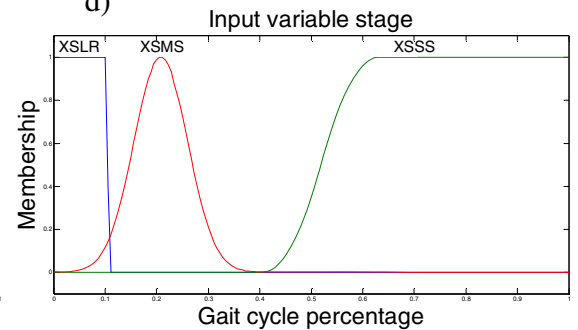

Fig. 3. Membership function definition for input variables. a) Knee, b) Hip, c) Time, d) Stage.

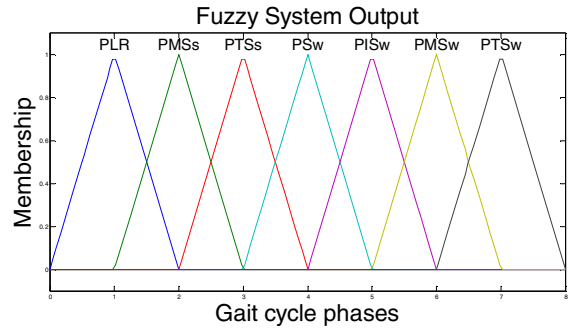

Fig. 4. Output membership function definition

The value of variable stage is located in the interval $L R$, meanwhile the variable time is located in the interval low. Inference using this information yields the system output loading response, $P_{L R}$. The same methodology was used to determine the other rules expressed next.

R1. If Hip is $x_{\text {hHigh }}$ AND Knee is $x_{k L o w}$ AND Stage is $x_{S L R}$ AND Time is $x_{\text {TLow }}$ Then Phase is $P_{L R}$

R2. If Hip is Not $x_{h H i g h}$ AND Knee is $x_{\text {kLow }}$ AND Stage is $x_{S M S}$ AND Time is $x_{\text {TLow }}$ Then Phase is $P_{M S S}$

R3. If Hip is $x_{\text {hMedium }}$ AND Knee is $x_{\text {kLow }}$ AND Stage is $x_{S M S}$ AND Time is $x_{\text {TLow }}$ Then Phase is $P_{M S S}$

R4. If Hip is $x_{\text {hLow }}$ AND Knee is $x_{\text {kLow }}$ AND Time is $x_{\text {TMedium }}$ Then Phase is $P_{\text {TSS }}$

R5. If Hip is $x_{\text {hLow }}$ AND Knee is Not $x_{\text {kLow }}$ AND Stage is $x_{S S S}$ AND Time is $x_{\text {TMedium }}$ Then Phase is $P_{S w}$

R6. If Hip is $x_{\text {hMedium }}$ AND Knee is Not $x_{\text {kLow }}$ AND Stage is $x_{S S S}$ AND Time is $x_{\text {TMedium }}$ Then Phase is $P_{I S w}$

R7. If Hip is $x_{\text {hHigh }}$ AND Knee is $x_{k H i g h}$ AND Stage is $x_{\text {SSS }}$ AND Time is $x_{\text {THigh }}$ Then Phase is $P_{M S w}$

R8. If Hip is $x_{h H i g h}$ AND Knee is $x_{k L o w}$ AND Stage is $x_{S S S}$ AND Time is $x_{\text {THigh }}$ Then Phase is $P_{T S w}$ 


\section{Experimental Results}

\subsection{Experimental Data}

The system was tested with two set of data representing two cases: normal gait and non-normal gait. The normal data is to verify that the system is able to detect the gait phases and the non-normal data to prove that the system does not detected the phases because they do not exist in these cases or they are misplaced. The different kinematic cases analyzed with the fuzzy system are as follows. Case 1 represents the kinematic of a patient with amputation below the knee. An average gait cycle is included in the cases 2, 4, 7, and 8. Case 7 includes average values in the upper bound and case 8 in the lower case. Case 3 is a person that presents spinal cord injury. Cases 5 and 6 correspond to patients with cerebral palsy.

Findings of the experiments using the previous cases indicate that the system was able to correctly detect the gait phases in the normal cases, meanwhile the system did not report some of the phases in the non-normal cases. Specific detail of each experiment is provided next.

Case1. In the case of the patient with amputation the kinematic tends to present a signal similar to a normal gait cycle, however the kinematic values are not always in the range of normal average values, as shown in Figure 5a. This observation can be visualized in Figure 5b, which presents the information of the occurrence of the gait phases. In a normal gait cycle the two traces must completely overlap. It can be noticed that the system detects phase 5, initial swing in the $84 \%$ of the gait cycle, when in this percentage the phase mid-swing should be detected. During the other gait cycle percentage the systems detects a normal behavior with respect phase occurrence and a small difference on the detection of phases 3, 4 and 7.

Case 2. First normal gait cycle. The signals corresponding to the knee and hip of this normal case are shown in Figure 6a. The result of the fuzzy system is illustrated in Figure $6 \mathrm{~b}$. The loading response phase is correctly detected in the percentage $0 \%$ to $10 \%$, as well as the mid-stance, $11 \%$ to $30 \%$. The terminal stance is located in the interval $31 \%$ to $49 \%$ and the pre-swing in the $50 \%$ to $59 \%$, which indicates a normal behavior because the average occurrence of this phase is between $50 \%-62 \%$. The initial swing is found in the range $63 \%-74 \%$, the mid-swing at $75 \%$ to $87 \%$ and the terminal swing at $88 \%-100 \%$. Comparing these results with the data in Table 1 , normal timing, it can be observed that the system reports very alike timing with just a few but not significant differences.

Case 3. Spinal injury case. The results of this case are presented in Figure $7 \mathrm{a}$ and $7 \mathrm{~b}$. It can be noticed from these figures that the system detected an anomalous behavior because the phase occurrence differences are large, in some occasions up to 6 percentage grades. Besides, an initial swing is detected in the terminal swing phase. Changes as the previously mentioned indicate a non-normal gait cycle. 
a)

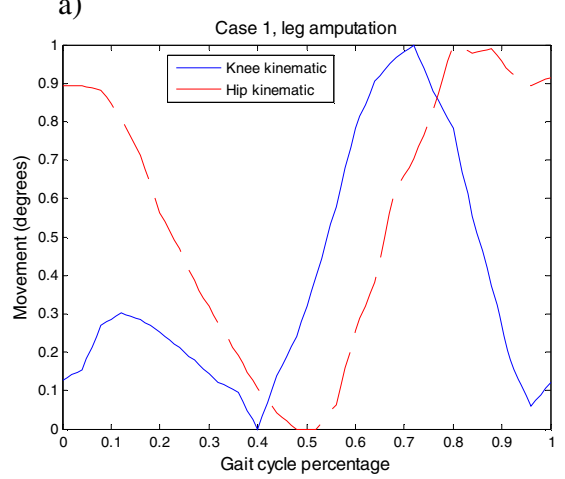

b)

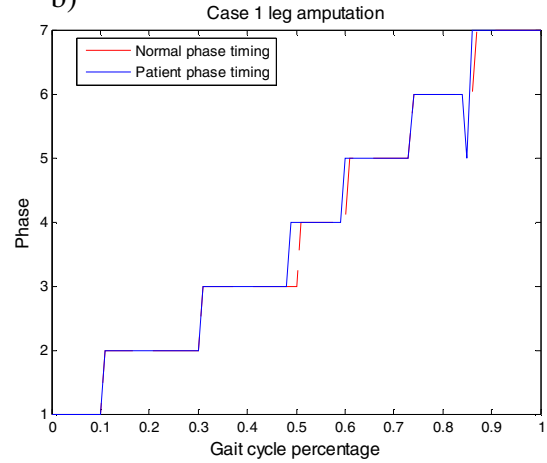

Fig. 5. Case 1 patient with leg amputation. a) Knee and hip kinematic. b) Phase detection, normal vs. system timing.

a)

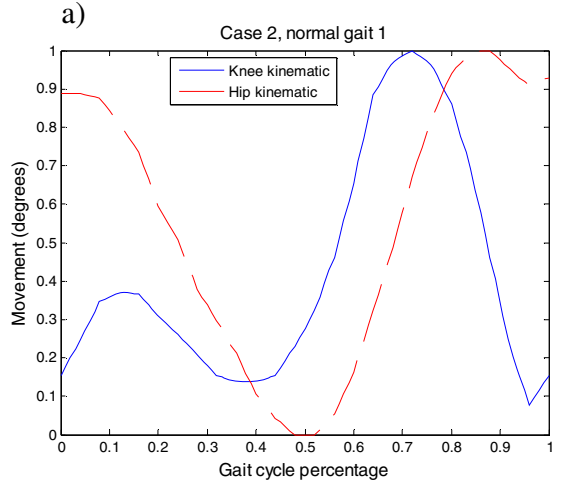

b)

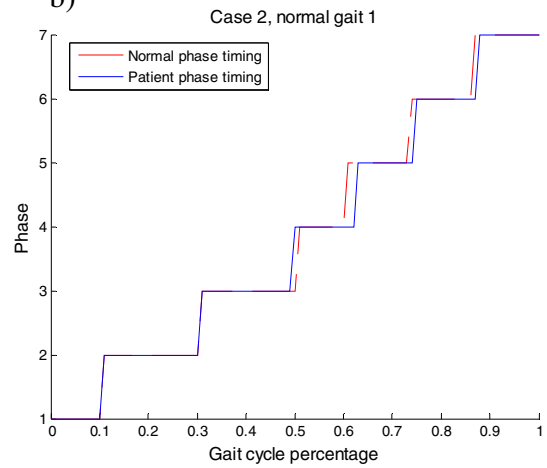

Fig. 6. Case 2 normal gait 1 . a) Knee and hip kinematic. b) Phase detection, normal vs. system timing.

a)

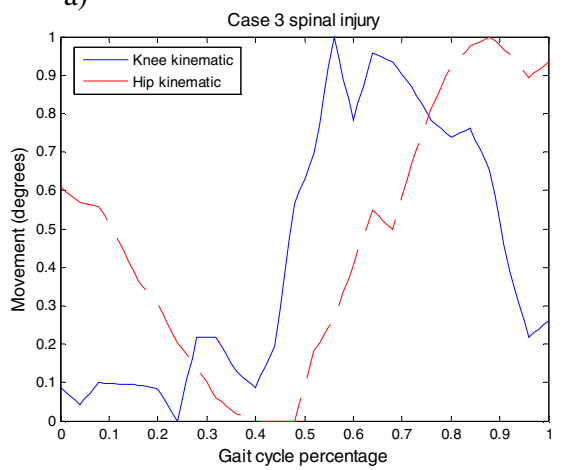

b)

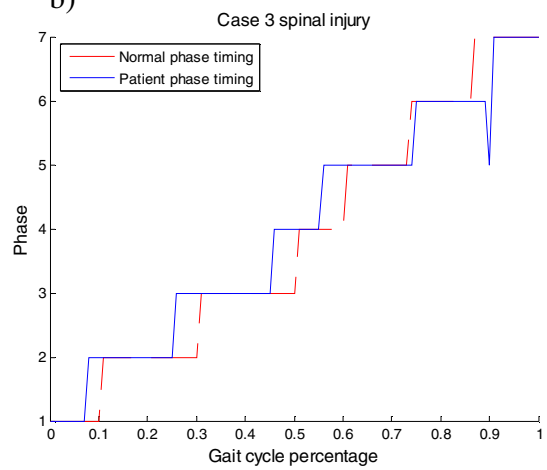

Fig. 7. Case 3 spinal injury. a) Knee and hip kinematic. b) Phase detection, normal vs. system timing. 
Case 4. Second normal gait cycle. This case and the cases 7 and 8 are incorporated into the experiments to warranty the robustness of the system to adequately detect normal gait cycle with normal variations. This case is not illustrated due to space constraints. The first three cycle phases, loading response, mid-stance and terminal stance are detected on time with the average gait cycle phases. Pre-swing, the four phase, is detected at $51 \%-62 \%$, the initial swing at $63 \%-74 \%$, mid-swing in the interval $75 \%-87 \%$ and finally the terminal swing in $88 \%$ to $100 \%$. The fuzzy system results present very similar time phase detection as for a normal gait cycle, being $2 \%$ the maximum difference.

Case 5. Cerebral palsy 1 , see Figure 8 . The phase detection timing generated by the system presents very high deviation as well as an inadequate sequence from the normal cycle. For example, the loading response phase is detected only during the $0 \%$ to $2 \%$ interval, when it must be identified from $0 \%$ to $10 \%$. Other observation is with regard the terminal stance phase, where an initial swing is reported.

a)

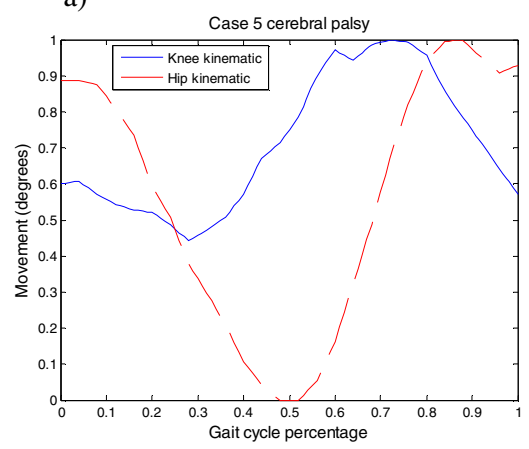

b)

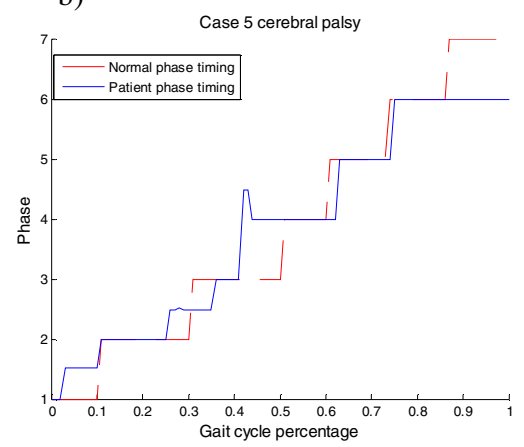

Fig. 8. Case 5 cerebral palsy 1. a) Knee and hip kinematic. b) Phase detection, normal vs. system timing.

Case 6. Cerebral palsy 2. The kinematic and phase detection results are reported in Figure 9. As in the first cerebral palsy case, it can be observed how phase occurrence does not follow the normal timing. The loading response phase, as in the previous case is only detected during the $0 \%$ to $2 \%$, besides and erroneous detection of a preswing phase during mid-stance.

Case 7. Normal gait cycle upper bound. This case corresponds to high average normal kinematic values. The kinematic is illustrated in Figure 10a and the phase detection results in Figure 10b. Results show that the first 3 phases are correctly detected following a normal phase timing. The fourth phase, pre-swing, was detected in $50 \%$ $62 \%$ which is considered correct. The initial swing is found in the $63 \%$ to $74 \%$, midswing at $75 \%-87 \%$ and the terminal swing at $88 \%-100 \%$, thus having a maximum deviation of $1 \%$ with respect the correct timing.

Case 8 . Normal gait cycle lower bound. This case covers the lower kinematic values for a normal gait cycle. This case is not illustrated due to space constraints. The phase loading response, mid-stance and terminal stance are detected at the correct timing. The other phases, pre-swing, initial swing, mid-swing and terminal swing 
a)

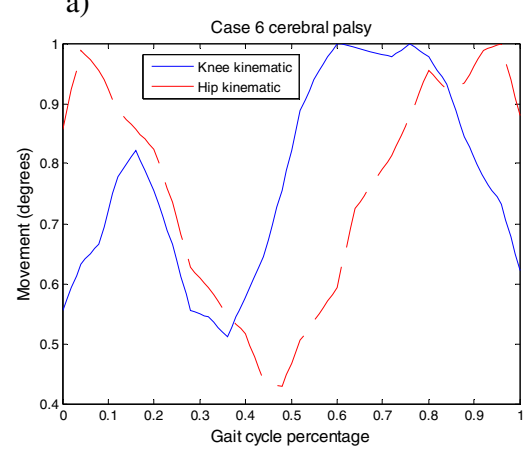

b)

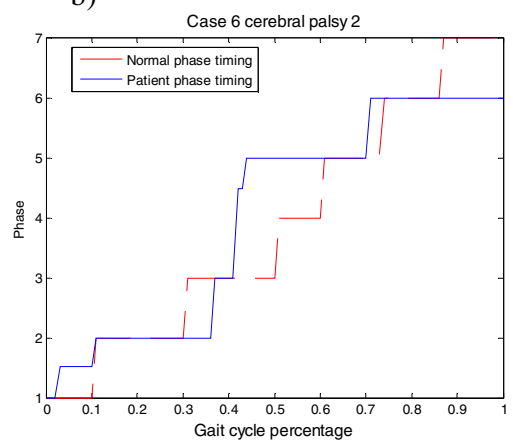

Fig. 9. Case 6 Cerebral palsy 2. a) Knee and hip kinematic. b) Phase detection, normal vs. system timing.

a)

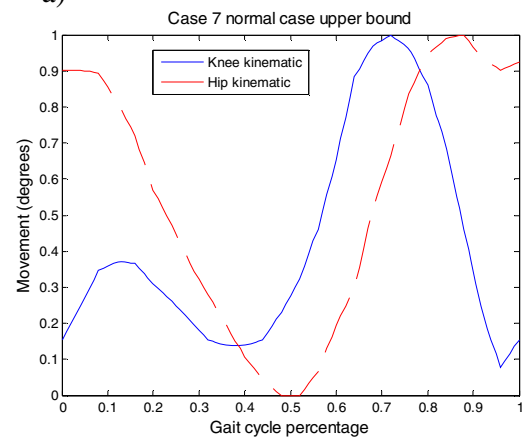

b)

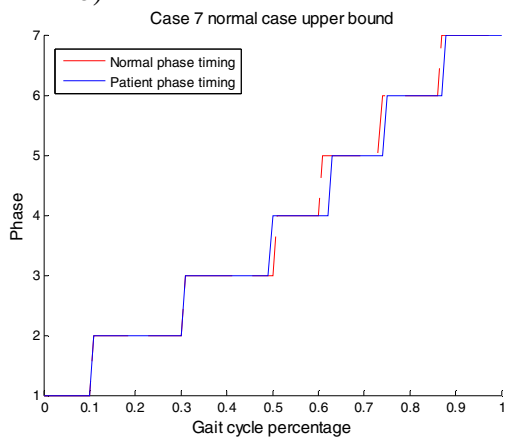

Fig. 10. Case 7 normal case upper bound. a) Knee and hip kinematic. b) Phase detection, normal vs. system timing.

were located at $51 \%-62 \%, 63 \%-74 \%, 75 \%-86 \%$ and $87 \%-100 \%$ respectively. Only the phases pre-swing and initial swing present a small time shift. The maximum phase shift was $2 \%$.

\section{$5 \quad$ Results and Conclusions}

The reported work proved that a fuzzy gait phase detection system is able to correctly detect the phases using only $2 \mathrm{D}$ information. The system was tested with non-normal as well as variation of normal gait cycle cases, in both circumstances the system showed acceptable results on the detection of the gait cycle phases for the normal cases. The maximum timing phase shift error generated was $2 \%$ for normal cases. In the non-normal cases the system reported misplaced phases as expected as a result of inadequate knee and hip kinematic due to the physical problems of the patients. Thus, it may be concluded that the proposed fuzzy system can be used to analyses gait kinematic by detecting gait phases in normal cycle and absences of them in non-normal 
cycles. This information may be considered for gait anomalies detections as well as therapeutic purposes.

Acknowledgements. The authors thanks to Fondo Mixto de Fomento a la Investigación Científica y Tecnológica CONACYT-Gobierno del Estado de Chihuahua and DGEST, by the support of this research under grant CHIH-2009-C02-125358 and CHI-MCIET-2011-103, respectively.

\section{References}

1. Perry, A.J.: Gait Analysis: Normal and Pathological Function. Slack, NJ (1992)

2. Senanayake, B.C.M., Senanayake, S.M.: Computational Intelligent Gait Phase Detection System to Identify Pathological Gait. IEEE Transactions on Information Technology in Biomedicine 14(5), 1173-1179 (2010)

3. Cardenas Maciel, C.S.L., Castillo, O., Aguilar, L.T.: Generation of Walking Periodic Motions for a Biped Robot Via Genetic Algorithms. Appl. Soft Comput. 11(8), 5306-5314 (2011)

4. Cardenas Maciel, D.S.L., Castillo, O., Aguilar, L.T., Castro, J.R.: A TS Fuzzy Logic Controller for Biped Robot Walking Based on Adaptive Network Fuzzy Inference System. In: Proceeding of the IJCNN, pp. 1-8 (2010)

5. Courtney, J., Paor, A.M.: A Monocular Marker-Free Gait Measurement System. IEEE Transactions on Neural Systems and Rehabilitation Engineering 18(4), 453-460 (2010)

6. Kirtley, F.C.: Clinical gayt analysis, http://www. clinicalgaitanalysis.com/

7. Lauer, G.R., Smith, B.T., Betz, R.R.: Application of a Neuro-fuzzy Network for Gait Event Detection Using Electromyography in the Child With Cerebral palsy. IEEE Transactions on Biomedical Engineering 52(9), 1532-1540 (2005)

8. Pappas, O.I., Popovic, M.R., Keller, T., Dietz, V., Morari, M.: A reliable gait phase detection system. IEEE Trans Neural Systems and Rehabilitation Engineering 9(2), 113-125 (2001)

9. Goffredo, I.M., Nixon, M., Carter, J.: 2D Markerless Gait Analysis. In: Proceedings of the 4th European Congress for Medical And Biomedical Engineering (2008)

10. Yuan, Q., Chen, I., Lee, S.P.: SLAC: 3D localization of human based on kinetic human movement capture. In: Proceedings of IEEE Robotics and Automation, pp. 848-853 (2011)

11. Arias-Enriquez, O.: Human Gait Analysis Based on 2D/Kinect Visual Perception and Diagnosis With Fuzzy Logic. Master thesis, Chihuahua Institute of Technology (2012)

12. Senanayake, C., Senanayake, S.M.: Evaluation of gait parameters for gait phase detection during walking. In: IEEE Proceedings of Multisensor Fusion and Integration for Intelligent Systems, Salt Lake City, UT, pp. 127-132 (2010)

13. MacDonald, C., Smith, D., Brower, R., Ceberio, M., Sarkodie-Gyan, T.: Determination of Human Gait Phase Using Fuzzy Inference. In: Proceedings of IEEE Rehabilitation Roboticsonal, pp. 661-665 (2007)

14. Chacon-Murguia, M.I., Sandoval-Rodriguez, R., Arias-Enriquez, O.: Human Gait Feature Extraction Including Kinematic Analysis Toward Robotic Power Assist. International Journal of Advanced Robotic Systems 9(68) (2012) 